\title{
Global Stable Disease in Lymph Nodes
}

National Cancer Institute

\section{Source}

National Cancer Institute. Global Stable Disease in Lymph Nodes. NCI Thesaurus. Code C159960.

Fails to attain the criteria for complete response, partial response, or progressive disease. 